\title{
Silencing ECHS1 attenuates the proliferation and induces the autophagy of hepatocellular carcinoma via impairing cell metabolism and activating AMPK
}

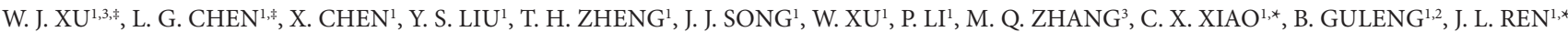

${ }^{1}$ Department of Gastroenterology, Zhongshan Hospital affiliated to Xiamen University, 201 Hubin South Road, Xiamen, Fujian Province, China, 361004; ${ }^{2}$ Faculty of Clinical Medicine, Medical College of Xiamen University, 168 University Road, Xiamen, Fujian Province, China, 361005; ${ }^{3}$ Department of Gastroenterology, 175 Hospital of PLA, affiliated Southeast Hospital to Xiamen University, Zhangzhou, Fujian Province, China, 363000

*Correspondence: xiaoxx@163.com,renjianl@xmu.edu.cn

${ }^{*}$ Contributed equally to this work.

Received March 19, 2015 / Accepted July 8, 2015

\begin{abstract}
Hepatocellular carcinoma (HCC) is among the most common cancers in the world with a low survival rate. Our previous study showed Short chain enoyl-CoA hydratase (ECHS1) could bind to HBsAg (HBs) and that ECHS1's localization in mitochondria induced HepG2 cell apoptosis. However, the role of the ECHS1 in energy metabolism and autophagy during hepatocellular carcinoma development remains undefined. We aimed to determine what ECHS1 does to energy metabolism and its effects on HCC progression. We performed CCK-8, EdU assays in hepatocellular carcinoma cell lines (HepG2 and HuH7) with stable ECHS1 knock-down. ATP and NADP+/NADPH levels were measured using an colorimetric assay. Our data demonstrated that ECHS1 silencing inhibited cell proliferation and induced autophagy. ECHS1 knockdown did not increase fatty acid synthesis, but decreased cellular ATP. This resulted in AMP-activated protein kinase (AMPK) activation and induced HCC cell autophagy. Our results showed that silencing ECHS1 to attenuate proliferation and induce autophagy may make it a novel cancer therapy target.
\end{abstract}

Key words: ECHS1, autophagy, hepatocellular carcinoma, cell metabolism, AMPK

HCC is one of the deadliest cancers with a five-year survival rate of less than 5 percent, and it is a complex and heterogeneous tumor with several genomic alterations $[1,2]$. Molecular targeted therapies have become novel high throughput technologies to treat hepatocellular carcinoma, such as sorafenib, a multikinase inhibitor with survival benefits for advanced hepatocellular carcinoma patients [3]. Our previous study showed ECHS1 could bind to HBs and that ECHS1's localization in mitochondria induced HepG2 cell apoptosis [4]. So, we suggested that ECHS1 may be applied as a potential therapeutic target during HCC treatment.

ECHS1 is purified from the human liver, and it is the second step in mitochondrial fatty acid $\beta$-oxidation (FAO). Mitochondrial FAO is a significant metabolic pathway that provides energy, especially under physiological conditions in which glucose as the primary energy source is reduced. Inherited mitochondrial FAO disorders form a metabolic disease class associated with high mortality rates [5]. ECHS1 deficiencies contributed to high-fat diet induced (HFD-induced) hepatic steatosis in rat models [6]. ECHS1 also regulated cellular ATP consumption/production and defense responses to ischemic stress [7]. When liver injuries or liver surgery was performed, they resulted in a sudden and massive proliferation of hepatocytes, and ECHS1 and other oxidative stress and fatty acid metabolism proteins were significantly enriched [8]. The liver regeneration process was impaired when fatty acid oxidation was inhibited by $\beta$-oxidation inhibitors [9].

Lipid and fatty acid metabolism plays an important role in cancer pathogenesis. fatty acid synthase(FAS) overexpression occurs in many cancer types and is frequently correlated with poor prognosis [10-13]. Researchers have tested inhibitors against this enzyme as anti-cancer drugs. During metabolic stress, FAO has a crucial role in cancer cell survival, and it sustains ATP levels and NADPH production $[14,15]$. Researchers also observed that NADPH availability was controlled by the LKB1-AMP K axis. 
AMPK is a highly conserved cellular energy status sensor comprised of one catalytic subunit $\alpha$ and two regulatory subunits $\beta$ and $\gamma$. Although the LKB1-AMPK axis is thought to be tumor suppressive, AMPK activity is essential for cancer cells to 'sense' stressful environments and respond by activating a catabolic switch that increases ATP and NADPH reserves [16]. AMPK switches on catabolic processes that provide alternative pathways to generate ATP while switching off anabolic pathways and other processes consuming ATP, thus acting to restore cellular energy homeostasis. It was activated by ATP depletion or glucose starvation, and researchers also previously thought AMPK activated autophagy by inactivating the mTOR complex- 1 and inhibiting cell proliferation $[17,18]$. Autophagy has an important role in cancer, and researchers proposed that inhibiting this cellular process contributes to HCC progression [19]. In this study, we aimed to determine what silencing ECHS1 does to energy metabolism and its effects on HCC progression.

\section{Materials and methods}

Cell culture. We purchased HepG2 and HuH7 cells from ATCC (commercially purchased from ATCC, Manassas, VA). We cultured cells in Dulbecco's Modified Eagle's Medium (DMEM) and supplemented them with 10\% fatal bovine serum (Life Technologies, Grand Island, NY), $100 \mathrm{U} / \mathrm{ml}$ penicillin, and $100 \mathrm{mg} / \mathrm{ml}$ streptomycin in a $37^{\circ} \mathrm{C}$ incubator with $5 \% \mathrm{CO}_{2}$.

Establishing stable ECHS1-knockdown cell lines. We designed two siRNAs to target ECHS1 and ligated the two siRNA target sequences (siECHS1-1: sense strand: 5'-GCCCATATCGTTTCATA GCTT-3'; antisense strand: 5'-AAGCTATGAAACGATATGGGC-3'; siECHS1-2: sense strand: 5'-GTAGATGAGATGTGACGAATT-3'; antisense strand: 5'-AATTCGTCACATCTCATCTAC-3') into control plasmids pu6 (pcPUR + U6-siRenilla) that targeted ECHS1. We transfected siECHS1 (pcPUR + U6-siECHS1) or pu6 (pcPUR +U6-siRenilla) into HepG2 and HuH7 cells with $2 \mathrm{ug} / \mathrm{ml}$ puromycin (Invitrogen, Carlsbad, CA) for two weeks and collected the puromycin-resistant colonies together. We examined ECHS1 expression using western blotting with an antibody against ECHS1 (experiments were repeated three times) to validate the constructs' efficiency at blocking the target gene.

Cell Counting Kit-8 (CCK8) assay. The two HCC cell lines were plated in 96-well plates at a density of 5,000 cells per well with $100 \mathrm{ul}$ of culture in DMEM medium. After adhesion, we removed the supernatant at $6,24,48$, and $72 \mathrm{~h}$ and added $100 \mathrm{ul}$ medium containing $10 \mathrm{ul} \mathrm{CCK8}$ (DoJinDo, Tokyo, Japan) to each well, incubating for another hour at $37{ }^{\otimes} \mathrm{C}$. We recorded the absorbance at $450 \mathrm{~nm}$. The detected OD was $450 \mathrm{~nm}$ in the two HCC cell lines in complete culture medium ( $4.5 \mathrm{~g} / \mathrm{L}$ D-Glucose) and low glucose culture medium (1.0 g/L D-Glucose). All experiments were independently repeated three times.
EdU cell proliferation assay. We cultured the cells in 8-well culture plates (BD Biosciences, Bedford, MA). After $24 \mathrm{~h}$, we added 5-ethynyl-20-deoxyuridine (EdU) $(100 \mathrm{mM})$ (CellLight EdU DNA Imaging Kit, Guangzhou RiboBio, China) and cultured the cells for an additional $2 \mathrm{~h}$. We then stained the cells according to the following protocol: we discarded the EdU medium mixture, added $4 \%$ paraformaldehyde to fix cells at room temperature for $30 \mathrm{~min}$, washed them with glycine $(2 \mathrm{mg} / \mathrm{ml})$ for $5 \mathrm{~min}$ in a shaker, added $0.5 \%$ Trion X-100 for $10 \mathrm{~min}$, washed them with PBS two times, added Apollo staining reaction buffer for $30 \mathrm{~min}$ while protecting from light, washed them with $0.5 \%$ Triton X-100 three times, stained them with Hoechst $33342(10 \mathrm{ug} / \mathrm{ml})$ for $30 \mathrm{~min}$ at room temperature, and washed them with PBS three times. Images were taken and visualized with fluorescence microscopy. EdU-positive cells were obtained from the fluorescent images.

Western blot analyses. We collected the cultured cells and lysed them with Mammalian Cell Lysis Reagent (Thermo Scientific, Rockford, IL). The proteins were separated using $8 \%, 10 \%, 12 \%$, or $15 \%$ sodium dodecyl sulfate polyacrylamide gel electrophoresis (SDS-PAGE) according its MW, and proteins were transferred to polyvinylidene difluoride (PVDF) membranes using semidry electro-blotting. The membranes were blocked in 5\% nonfat milk, dissolved in TBST for $1 \mathrm{~h}$ at room temperature, and incubated with the primary antibody overnight at $4{ }^{\circ} \mathrm{C}$. The next day, we washed the secondary antibodies conjugated with horseradish peroxidase (HRP) and incubated them for $1 \mathrm{~h}$ at room temperature. Then, we detected the immunoreaction using the Enhanced Chemiluminescence Kit (Amersham Pharmacia, Piscataway, NJ) and quantified it using densitometry. We purchased the antibodies against tubulin, Fatty Acid and Lipid Metabolism Antibody Sampler Kit, and AMPK and Acetyl-CoA carboxylase (ACC) Antibody Sampler Kit from Cell Signaling Technologies (Boston, MA, USA), ECHS1 from Abcam (1:1000 dilution, Cambridge, MA), and protein A/B light chain 3-II (LC3A/B) from Santa Cruz (1:1000 dilution, Santa Cruz, CA).

Immunocytochemistry. The two HCC cell lines were incubated in 8-well Glass at a density of 5,000 cells per well with $500 \mathrm{ul}$ low glucose culture medium (1.0 g/L D-Glucose), cultured $24 \mathrm{~h}$, then removed, washed with PBS, and fixed in $4 \%$ paraformaldehyde for $15 \mathrm{~min}$ at room temperature. They were perforated with $0.3 \%$ Triton X-100 for $10 \mathrm{~min}$, blocked with 5\% BSA in PBS, and incubated with mouse monoclonal anti-LC3A/B (1:1000 dilution, Santa Cruz, CA) for $1 \mathrm{~h}$. After being washed, cells were incubated with various fluorochromeconjugated secondary antibodies for $1 \mathrm{~h}$. We performed cell imaging using an Only Broadband Confocal Leica TCS SP5 II (Leica Microsystems GmbH, Wetzlar, Germany) and analyzed the images with MetaMorph/MetaFluor version 7.0 software (Leica Microsystems GmbH, Wetzlar, Germany).

Colorimetric measurements (ATP, NADP+/NADPH). We measured cellular ATP levels using the ATP colorimetric assay kit (Biovion, San Francisco, USA) according to the manufacturer's protocol. We lysed $1.5 \times 10^{6}$ cells in $100 \mathrm{ul}$ 


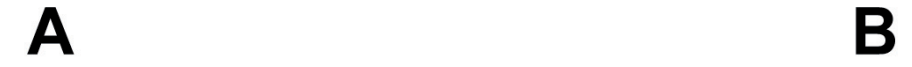

Normal
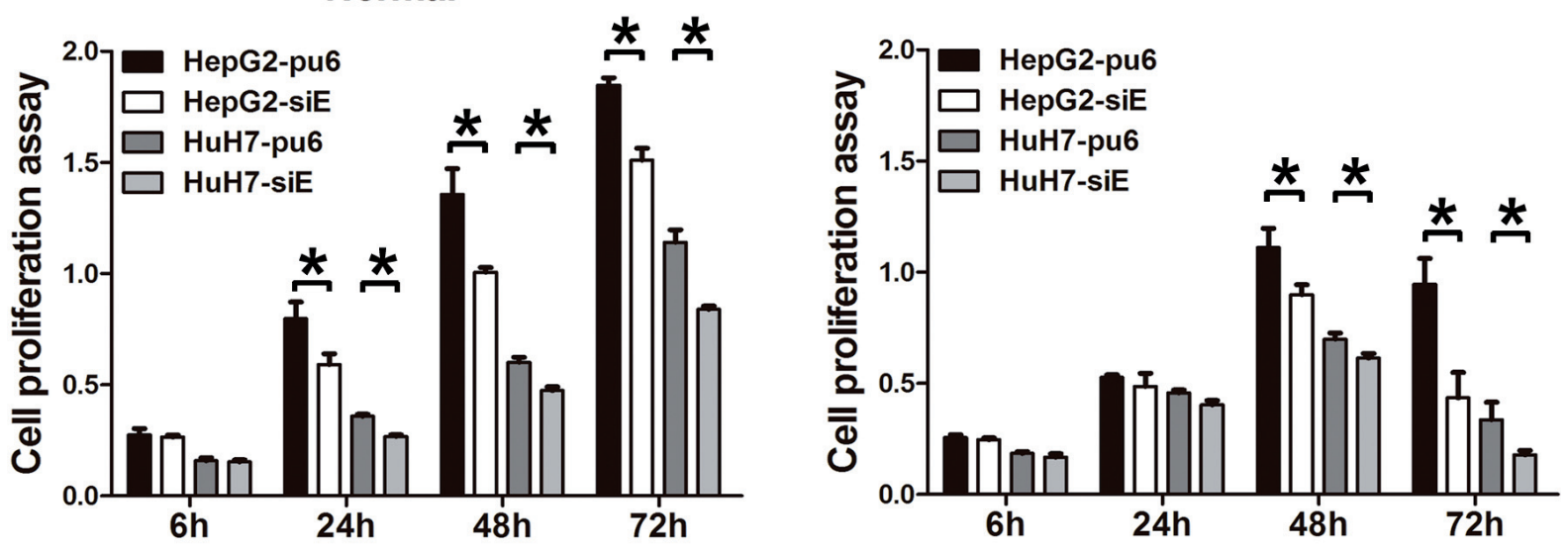

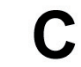

Normal
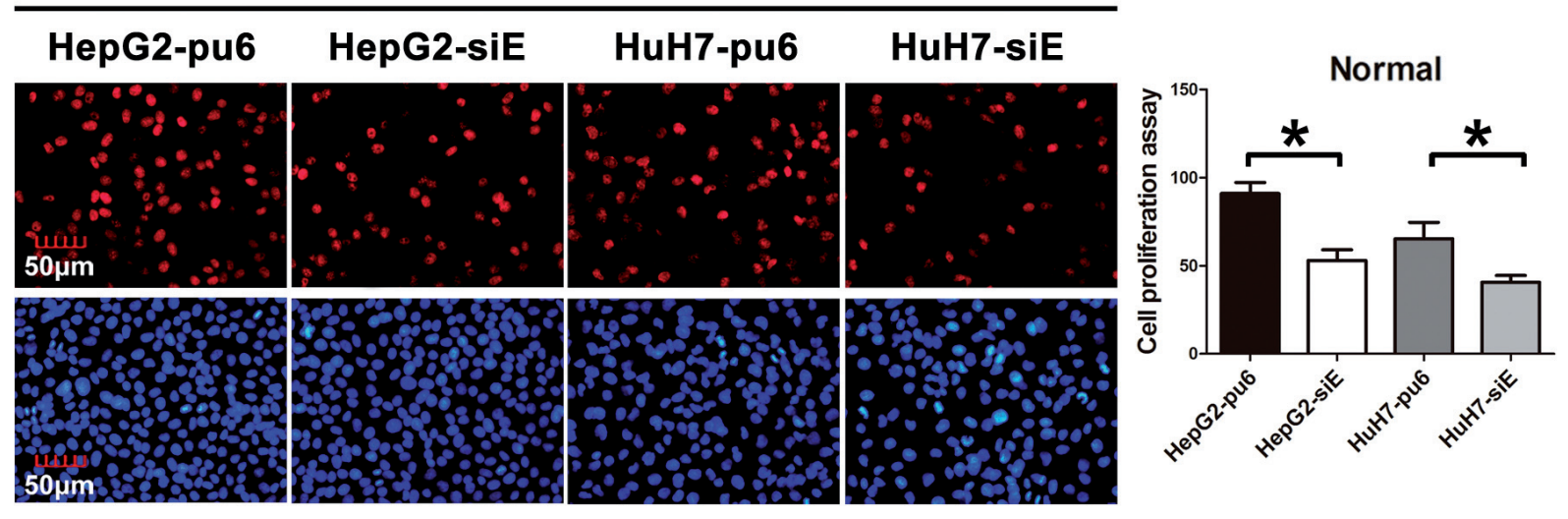

D

\section{Low Glu}
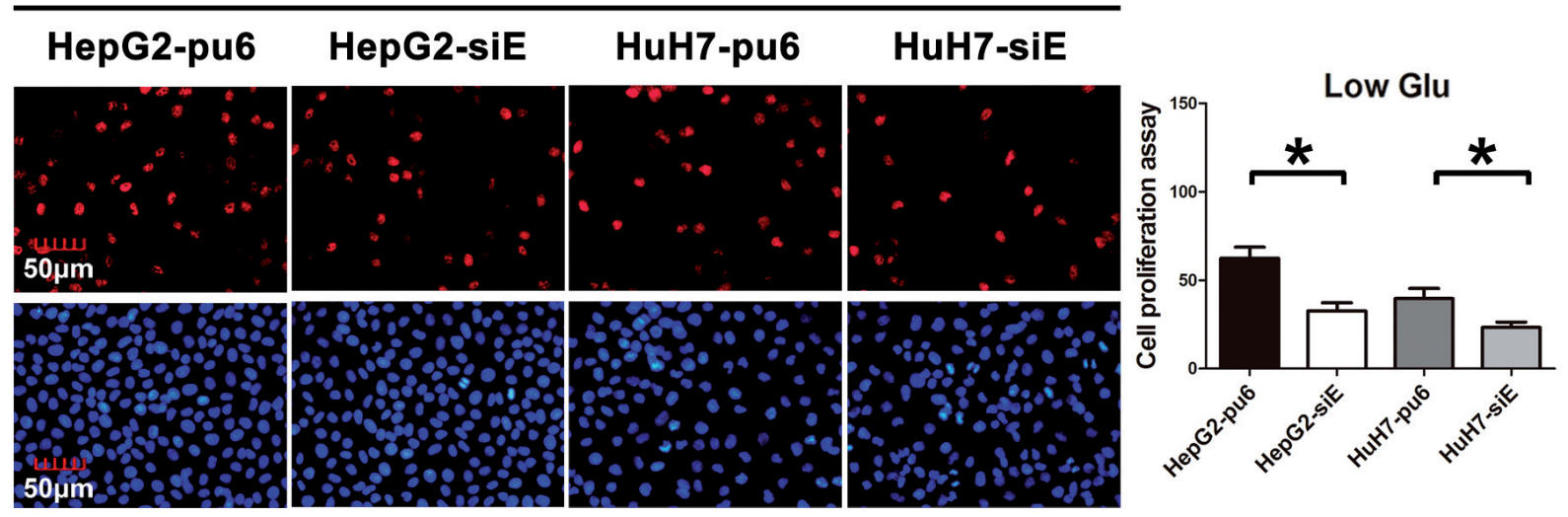

Figure 1. The cell proliferation assay revealed that HCC cell proliferation was inhibited by silencing endogenous ECHS1.

HepG2-pu6, HepG2-siECHS1, HuH7-pu6, and HuH7-siECHS1 cells were cultured in complete medium (4.5 g/L D-Glucose) (A, C) and low glucose medium (1.0 g/L D-Glucose) (B, D). Cell proliferation ability (OD value) was detected using a CCK-8 kit (A, B) and EdU cell proliferation assay (red color in C, D), Hoechst 33342 (blue color). Data are presented as the means \pm SD of three independent experiments. ${ }^{\star} P<0.05$. 
ATP buffer and deproteinized the cell lysate using a spin column. We added $35 \mathrm{ul}$ of the sample to a 96-well plate and adjusted the volume to $50 \mathrm{ul} /$ well with an ATP assay buffer. We prepared the standard curve and added $50 \mathrm{ul}$ reaction mix to each well, incubated it at room temperature for $30 \mathrm{~min}$ while protecting it from light, and then measured the absorbance (OD $570 \mathrm{~nm}$ ).

We measured NADP+/NADPH using a colorimetric kit (Biovion, San Francisco, USA). We extracted $10^{5}$ cells with $200 \mu \mathrm{l}$ of NADP/NADPH extraction buffer for each assay and set the standard curve as the kit specified. We transferred $50 \mu \mathrm{l}$ of the extracted samples into a labeled 96-well plate in duplicate to detect the total NADP+/NADPH (NADPt). We heated $200 \mathrm{ul}$ of the samples to $60^{\circ} \mathrm{C}$ for $30 \mathrm{~min}$ in a water bath to detect NADPH only, and added $100 \mu$ of the a NADP cycling mix (NADP Cycling Buffer Mix: $98 \mu$ l, NADP Cycling Enzyme Mix: $2 \mu \mathrm{l}$ ) into each well. We incubated the plate at room temperature for $5 \mathrm{~min}$ to convert NADP to NADPH and added $10 \mu \mathrm{l}$ NADPH developer into each well. We let the reaction develop for 1 to $4 \mathrm{~h}$ and read the plate at OD $450 \mathrm{~nm}$. We calculated the NADP+/NADPH ratio as: (NADPt - NADPH) / NADPH.

Statistical analyses. Statistical analysis was performed using SPSS 17.0 software (SPSS Inc., Chicago, IL). Data between two groups were compared using the Student's t-test. All values are expressed as the means \pm standard deviation (SD), and $P<0.05$ was considered to be statistically significant. Graphs were generated using GraphPad Prism 5.0 (GraphPad Software Inc., La Jolla, CA).

\section{Results}

Silencing endogenous ECHS1 attenuated cell proliferation in HepG2 and HuH7 cells. To explore the ECHS1 gene's function in HCC cells, we established two stable ECHS1interference cell lines (HepG2-siECHS1, HuH7-siECHS1) and their control cells (HepG2-pu6, HuH7-pu6). We confirmed our comparisons between ECHS1 knockdown cells and control cells through western blotting (Fig. 4A). ECHS1 played an important role in fatty acid $\beta$-oxidation, and free fatty acid (FFA) become more significant when glucose decreased. We examined whether knocking down ECHS1 expression had any effect on cell growth in complete medium ( $4.5 \mathrm{~g} / \mathrm{L} \mathrm{D}$-Glucose) and low glucose medium (1.0 g/L D-Glucose). The CCK-8 kit and EdU cell proliferation assay showed that silencing ECHS1 inhibited HCC cell proliferation, evidently with lower glucose compared to control cells (Fig. 1).

ECHS1 knockdown accelerated ATP and NADPH depletion and increased the NADP+/NADPH ratio. ATP is the primary energy currency of living systems. It is formed exclusively in mitochondria, and various genetic diseases and environmental changes effect ATP formation in the mitochondria. NADP+/NADPH was primarily used in anabolic biosynthetic reactions such as fatty acid synthesis and thus utilized significant amounts of energy. We assessed ECHS1's impact on cellular bioenergetics by changes in ATP contents and NADPH. Figures 2A, E, and G show that ECHS1 knockdown decreased cellular ATP and NADPH and increased the $\mathrm{NADP}+\mathrm{NADPH}$ ratio, and these effects were exaggerated in glucose-limited conditions (Fig. 2B, F, and H). Furthermore, we overexpressed the ECHS1 in stable konckdown ECHS1 of HepG2 and HuH7, We observed that the level of cellular ATP was increased both in normal and low glucose (Fig. 2C and D).

Silencing ECHS1 inhibited fatty acid metabolism and activated AMPK. ECHS1 is vital in fatty acid $\beta$-oxidation, and in this study we showed that silencing ECHS1 decreased ATP and NADPH levels. Furthermore, we investigated the protein alterations in fatty acid metabolism and AMPK. Figures 3A and $B$ showed that the long chain acyl-CoA synthetase (ACSL) enzyme, which catalyzes cellular ligation of free fatty acids to co-enzyme-A to form fatty acyl-CoA, was down-regulated as compared to control cells. Expressions of ATP-citrate lyase (ACL), phosphorylation of ATP-citrate lyase (P-ACL), and fatty acid synthase (FAS), which are proteins that catalyze fatty acid synthesis, were also decreased both in HepG2siECHS1 and HuH7-siECHS1 cells compared to control cells. The decreases were more obvious in low glucose conditions, suggesting that fatty acid synthesis was also inhibited. We also found that the phosphorylation of AMPK and ACC were increased in ECHS1-knockdown cells, suggesting that fatty acid synthesis was inhibited after ECHS1 silencing.

ECHS1 knockdown induced autophagy. Autophagy plays an important role in cell responses to stress [20]. Microtubuleassociated LC3-II protein was specifically associated with autophagosome membranes during the autophagy process and was an autophagy marker [21,22]. Immunoblot analyses indicated that LC3-II was significantly up-regulated in ECHS1-knockdown cells, whether in complete medium or glucose-limited medium (Fig. 4B and C). Consistent with the western blot analysis, confocal microscopy showed that LC3 proteins were also accumulated in HepG2-siECHS1 and HuH7-siECHS1 cells (Fig. 4D), and LC3 fusion protein fluorescence accumulated much more under glucose-limited conditions (Fig. 4D).

\section{Discussion}

In this study, we clearly demonstrated that silencing ECHS1 in HCC cells not only inhibited proliferation but also induced autophagy via impairing energy metabolism and activating AMPK. This result was not only found in complete medium $(4.5 \mathrm{~g} / \mathrm{L}$ D-Glucose $)$, but also in low glucose medium $(1.0 \mathrm{~g} / \mathrm{L}$ D-Glucose). Furthermore, we revealed that the primary energy of ATP and NADPH was decreased, these effects were exaggerated in glucose-limited conditions, and the fatty acid metabolism (including FAO and FAS) inhibited after silencing ECHS1 in HCC, different from normal hepatic cells. As previous studies reported, silencing ECHS1 in normal hepatic cells decreased FAO and led to accumulated fatty acid, resulting in 
A Normal

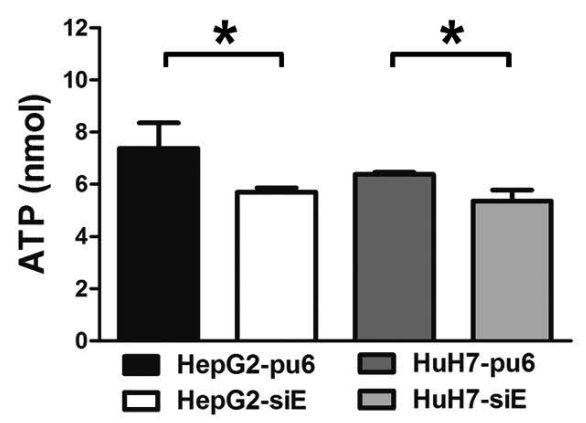

C
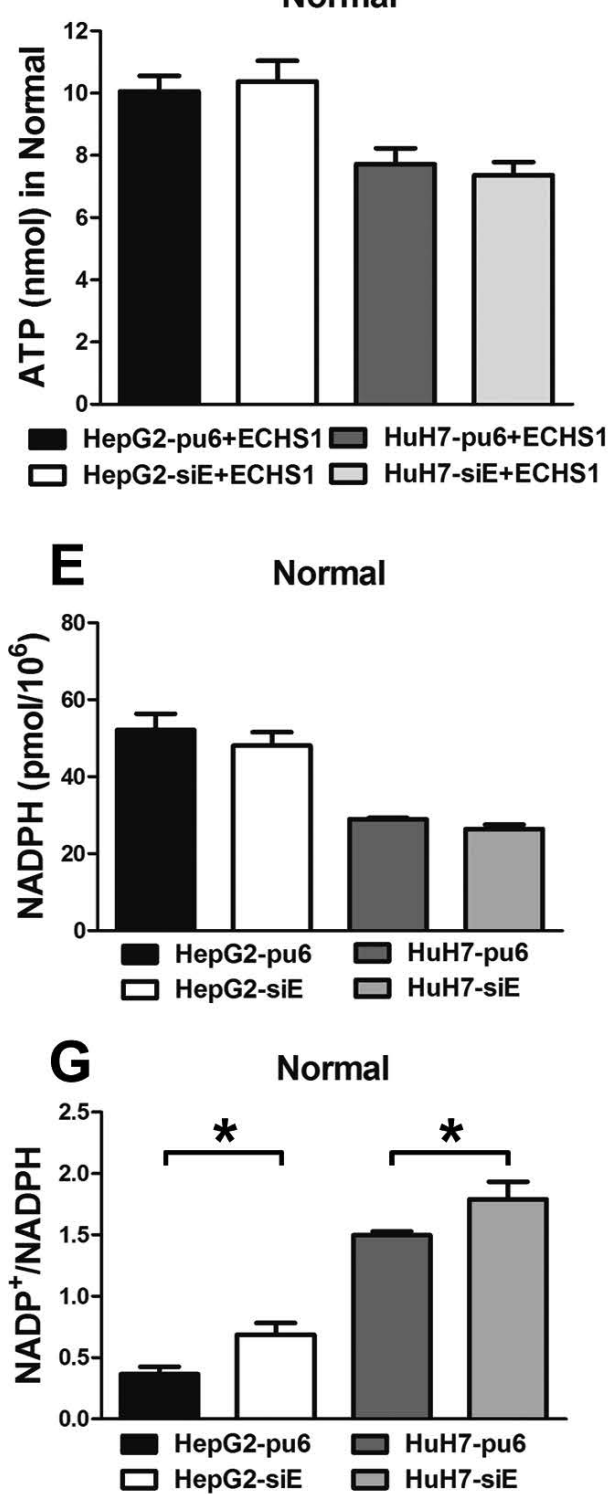

B

Low Glu
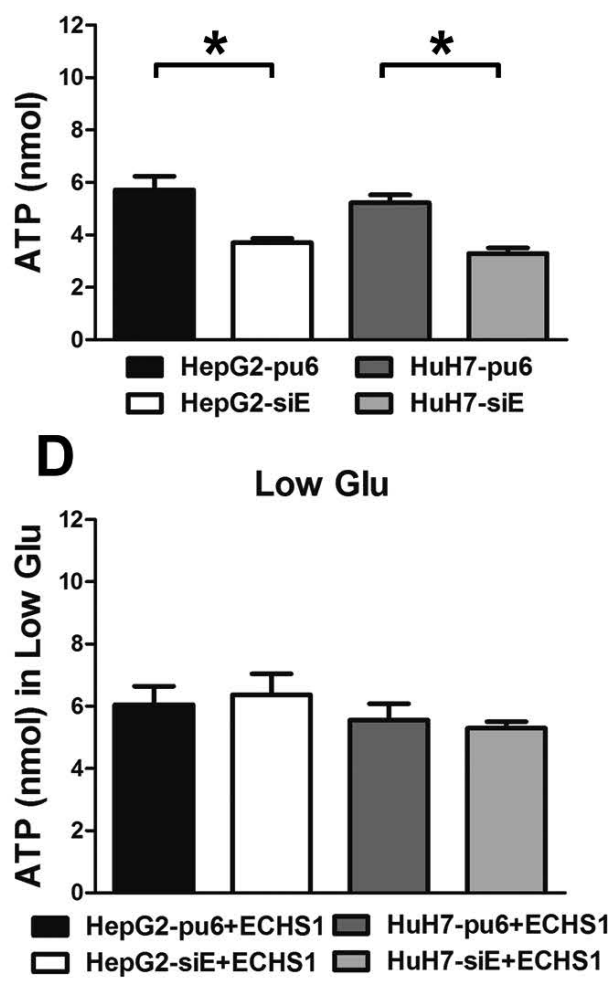

$\mathbf{F}$
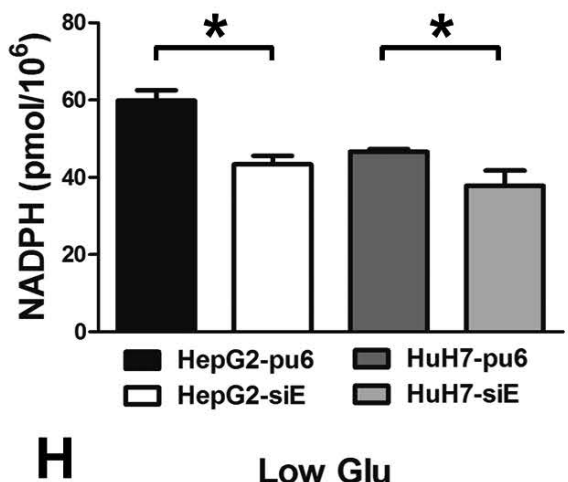

Low Glu

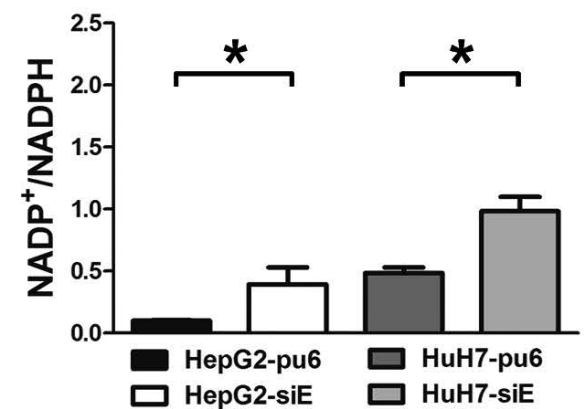

Figure 2. ATP and NADPH production decreased after ECHS1 silencing.

The ATP and NADPH levels were detected in HepG2-siECHS1, HuH7-siECHS1 and their control cells in normal medium (A, E), and ATP and NADPH were significantly decreased in low glucose medium (B, F). ATP was detected in normal and low glucose after overexpression ECHS1 (C, D). The NADP+/ NADPH ratio was higher in HepG2-siECHS1 and HuH7-siECHS1 cells than control cells $(\mathrm{G}, \mathrm{H}) .{ }^{*} \mathbf{P}<\mathbf{0 . 0 5}$. 

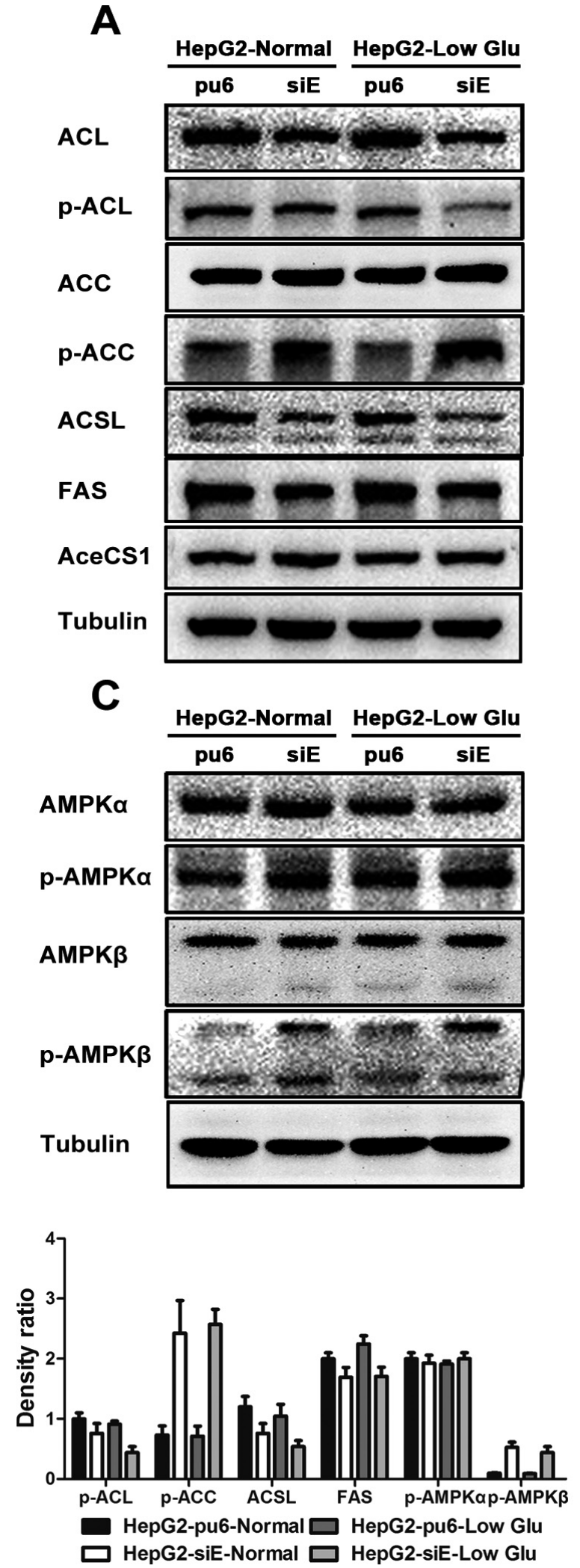
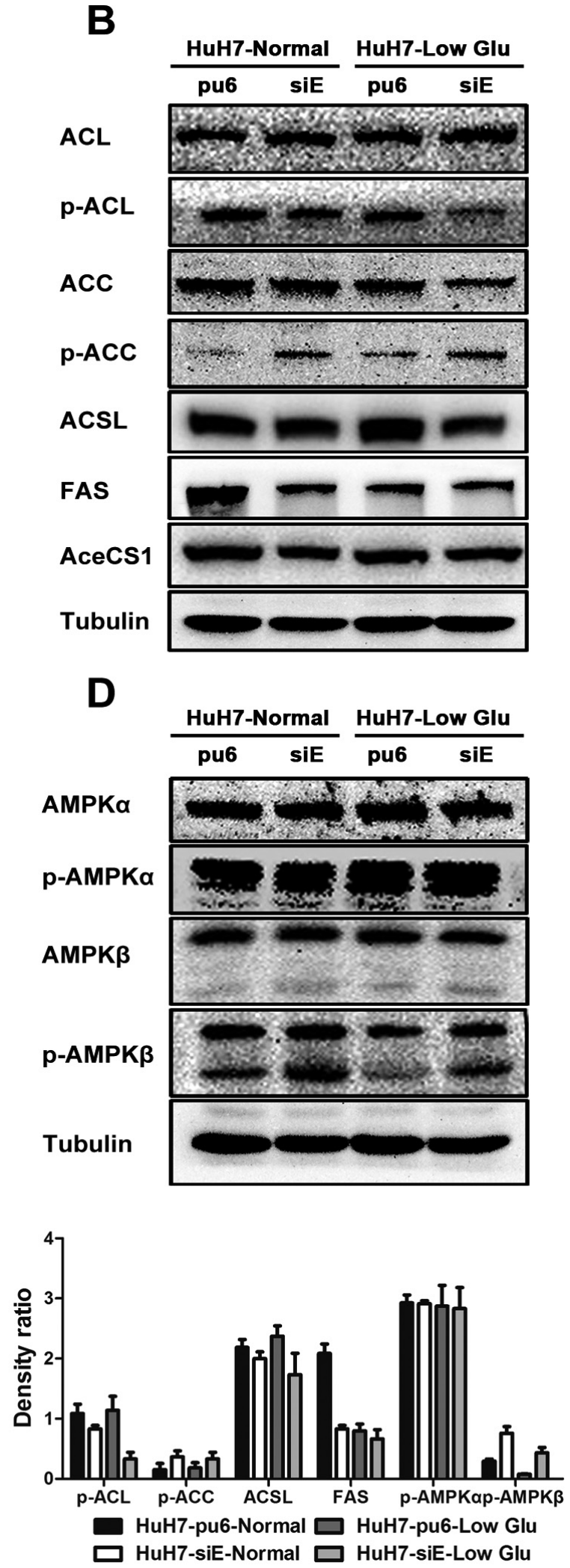

Figure 3. Western blot analysis of AMPK protein and fatty acid metabolism in HCC cells.

The western blot showed that ACL, P-ACL, FAS, and ACSL expressions were down-regulated and P-ACC expression was up-regulated as a result of silencing ECHS1 $(\mathrm{A}, \mathrm{B})$. We also found that P-AMPK $\beta$ was expressed higher than in controls $(\mathrm{C}, \mathrm{D})$. 


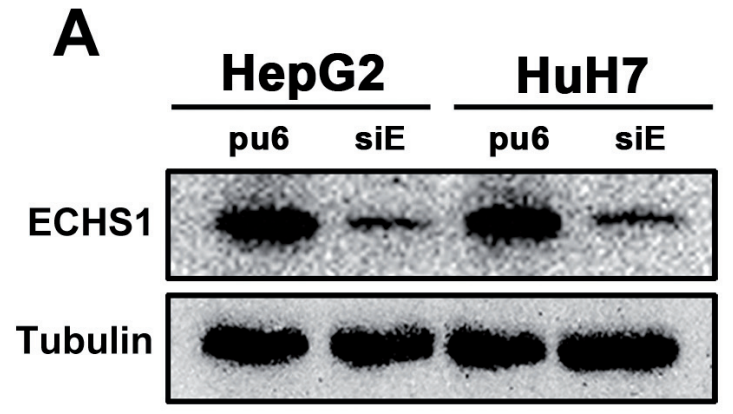

C

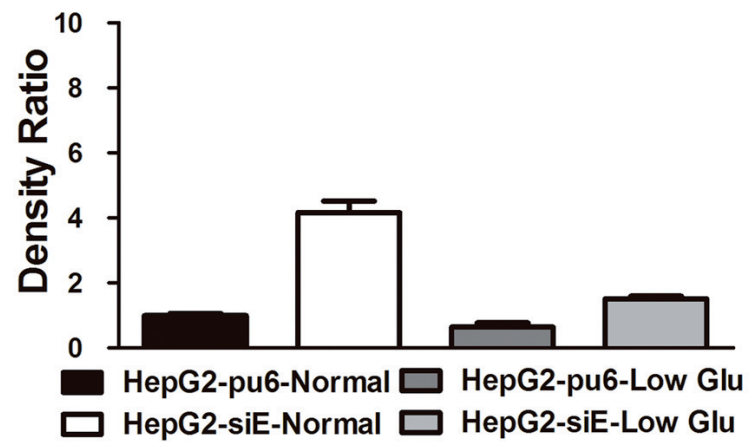

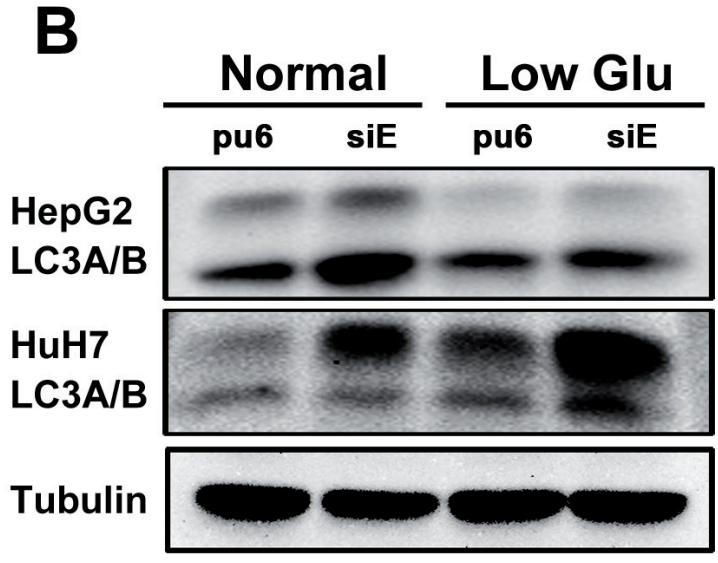

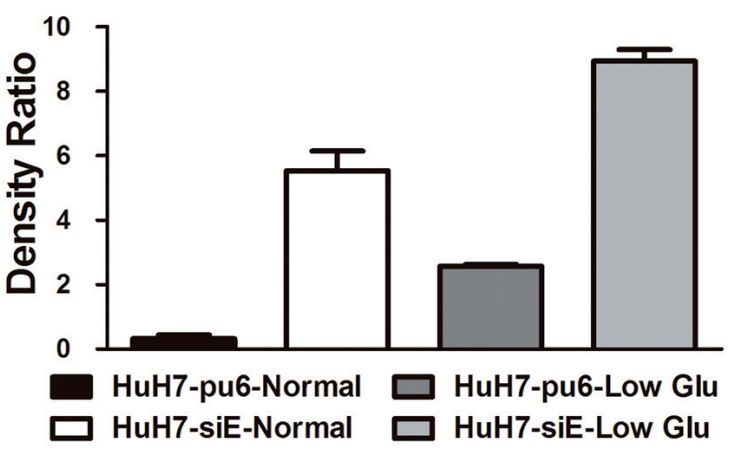

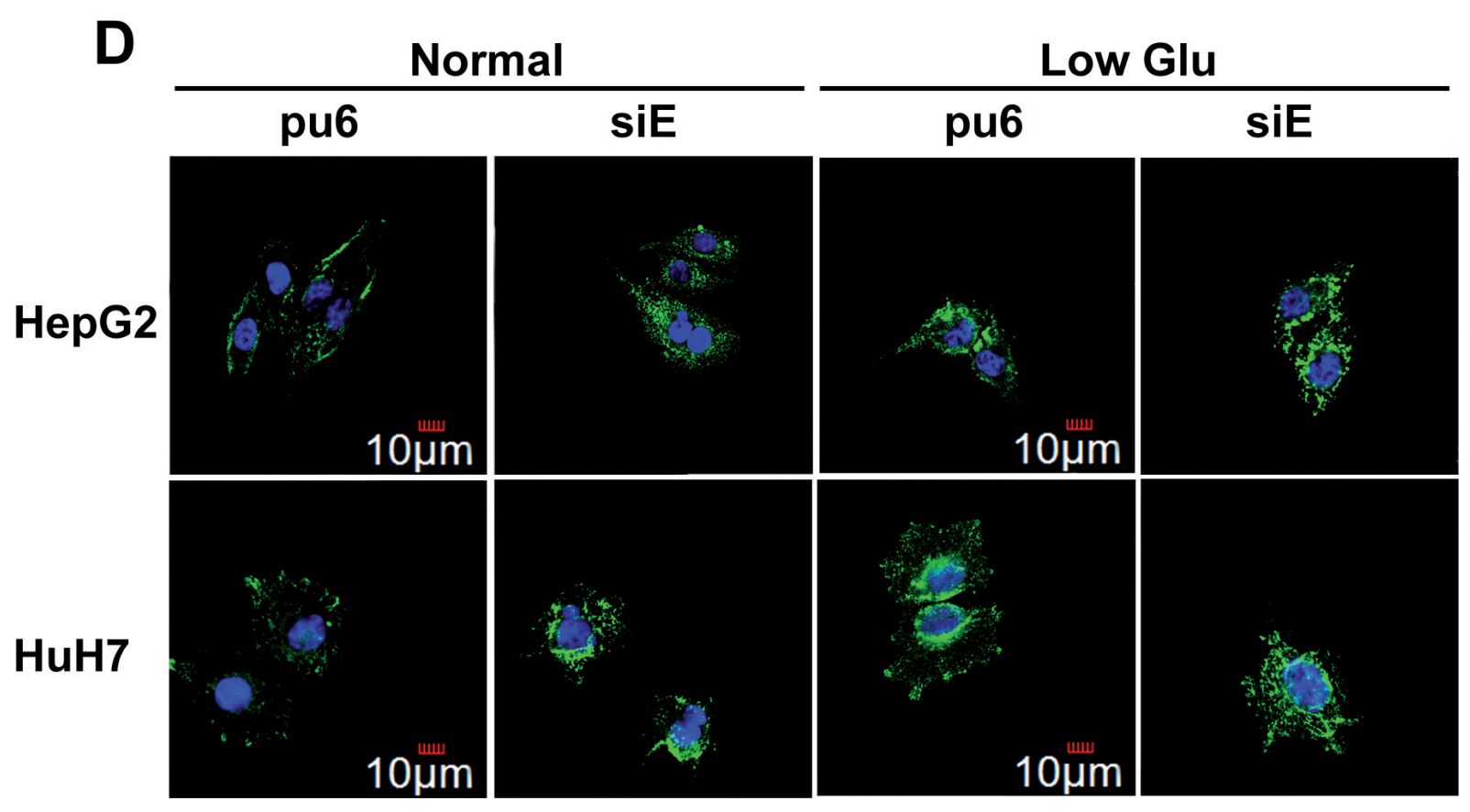

Figure 4. Silencing ECHS1 induced autophagy in HCC cells.

Western blots showing the levels of ECHS1 in the stable cells lines of HepG2-siECHS1 and Huh-7-siECHS1 (A). Western blot analysis of LC3-II accumulated in HepG2-siECHS1 and Huh-7-siECHS1 cells compared to control cells (B). Pixel densities of LC3 proteins from the stable cells lines of HepG2-siECHS1 and Huh-7-siECHS1 (C). Confocal microscopy of LC3 (green color), Hoechst 33342 (blue) puncta in HCC following stale ECHS1 transfection. The puncta accumulated significantly when incubated with low glucose medium (D). 
a fatty liver and hepatic steatosis. We propose that this might have been due to hepatoma carcinoma cells dividing more frequently than normal hepatic cells. This would imply that hepatoma carcinoma cells would have a higher energy demand for cellular survival, and would therefore lack energy to store in fatty tissues. We also suggested other pathways existed that regulate energy homeostasis, which would compensate for the energy shortage after silencing ECHS1 in HCC.

Altered metabolic activity is crucial for supporting uncontrolled proliferation, growth-inhibitory signal evasion, cell migration, and the dissemination of metastatic cells into distant tissues. In addition to glucose and glutamine metabolism, the increased biosynthesis of macromolecules, particularly lipids, has been recognized as a metabolic reprogramming component in cancer cells [23]. In vivo distribution of energy metabolites, including lipids and lipoproteins, significantly affected tumor cell survival and cancer development. It also impaired the viability of cancer cells, which could provide new strategies for cancer treatment. Anti-metabolites like metformin and pemetrexed, which activate AMPK-mediated inhibition of glycolysis and purine metabolism, are being widely investigated for cancer prevention and treatment strategies [24-26].

AMPK is a master regulator of metabolic homeostasis by sensing cellular energy status [27]. As a principal sensor, it switches off anabolic pathways such as fatty acids, triglyceride, cholesterol, and protein synthesis, and switches on catabolic pathways such as fatty acid oxidation and glycolysis $[18,28]$. We suggested AMPK activation suppressed proliferation cell via p53-p21 axis up-regulation and TSC2-mTOR regulation [29] or activated by AXIN-AMPK-LKB1 Complex [30]. AMPK also reduced FAS and ACC expressions, resulting in suppressed proliferation in prostate-cancer cells [31]. This may explain why silencing ECHS1 caused lower expressions of the fatty acid synthesis proteins FAS and ACC (Fig. 3A-B). It demonstrated that silencing ECHS1 impaired metabolism via activating AMPK to maintain metabolic homeostasis (Fig. 3C). Furthermore, inhibiting ACL suppressed tumor growth [32], so we suggested that the ECHS1-knockdown not only inhibited cell proliferation by activating AMPK, but may also result in inhibited ACL and P-ACL expressions.

During nutrient and metabolic stress, AMPK was activated via phosphorylation by LKB1, thus providing starved cells with nutrients by inducing autophagy [33]. Autophagy was quantified by measuring GFP-LC3 (or LC3 by immunofluorescence) and monitoring LC3-II using western blot. Figure 4 showed many more LC3 dots were detected in silenced ECHS1 cells than controls in a regular medium, and the number became much larger in low glucose medium. Autophagy serves as a critical adaptive response to starvation (amino acid and nutrient deprivation) and metabolic stress by recycling energy and nutrients [34], so we supposed silencing ECHS1 indirectly activated AMPK by inhibiting fatty acid synthesis and inducing autophagy to rescue cells from the deadly energy imbalance.
In conclusion, ECHS1 knockdown attenuated HCC proliferation via impairing cell metabolism and inducing autophagy, and a new study identified some small autophagy-inducing molecules that may prove useful in therapeutic applications [35-37]. Combined with the study we had done previously, we conclude that ECHS1 acted as a novel target for treating hepatocellular carcinoma.

Acknowledgments: This study was supported by National Natural Science Foundation of China (No. 81225025 \& 91229201), Ministry of Health Foundation for State Key Clinical Department, 863 and 973 programs (2012AA02A201\& 2013CB944903) in China.

\section{References}

1] JEMAL A, BRAY F, CENTER MM, FERLAY J, WARD E, et al. Global cancer statistics. CA Cancer J Clin 2011; 61: 69-90. http://dx.doi.org/10.3322/caac.20107

[2] PARKIN DM, BRAY F, FERLAY J, PISANI P. Global cancer statistics, 2002. CA Cancer J Clin 2005; 55: 74-108. http:// dx.doi.org/10.3322/canjclin.55.2.74

[3] LLOVET JM, BRUIX J. Molecular targeted therapies in hepatocellular carcinoma. Hepatology 2008; 48: 1312-1327. http:// dx.doi.org/10.1002/hep. 22506

[4] XIAO CX, YANG XN, HUANG QW, ZHANG YQ, LIN BY, et al. ECHS1 acts as a novel HBsAg-binding protein enhancing apoptosis through the mitochondrial pathway in HepG2 cells. Cancer Lett 2013; 330: 67-73. http://dx.doi.org/10.1016/j. canlet.2012.11.030

[5] JANSSEN U, DAVIS EM, LE BEAU MM, STOFFEL W. Human mitochondrial enoyl-CoA hydratase gene (ECHS1): structural organization and assignment to chromosome 10q26.2-q26.3. Genomics 1997; 40: 470-475. http://dx.doi. org/10.1006/geno.1996.4597

[6] ZHANG X, YANG J, GUO Y, YE H, YU C, et al. Functional proteomic analysis of nonalcoholic fatty liver disease in rat models: enoyl-coenzyme a hydratase down-regulation exacerbates hepatic steatosis. Hepatology 2010; 51: 1190-1199. http://dx.doi.org/10.1002/hep.23486

[7] ZHU SG, XI L, KUKREJA RC. Type 2 diabetic obese $\mathrm{db} / \mathrm{db}$ mice are refractory to myocardial ischaemic post-conditioning in vivo: potential role for Hsp20, F1-ATPase delta and Echs1. J Cell Mol Med 2012; 16: 950-958. http://dx.doi.org/10.1111/ j.1582-4934.2011.01376.x

[8] SEVERINO V, LOCKER J, LEDDA-COLUMBANO GM, COLUMBANO A, PARENTE A, et al. Proteomic characterization of early changes induced by triiodothyronine in rat liver. J Proteome Res 2011; 10: 3212-3224. http://dx.doi. org/10.1021/pr200244f

[9] NAKATANI T, OZAWA K, ASANO M, UKIKUSA M, KAMIYAMA Y, et al. Differences in predominant energy substrate in relation to the resected hepatic mass in the phase immediately after hepatectomy. J Lab Clin Med 1981; 97: 887-898.

[10] ZHU X, QIN X, FEI M, HOU W, GRESHOCK J, et al. Combined Phosphatase and Tensin Homolog (PTEN) Loss and Fatty Acid Synthase (FAS) Overexpression Worsens the Prognosis of Chinese Patients with Hepatocellular Carcinoma. 
Int J Mol Sci 2012; 13: 9980-9991. http://dx.doi.org/10.3390/ ijms13089980

[11] HORIGUCHI A, ASANO T, ITO K, SUMITOMO M, HAYAKAWA M. Fatty acid synthase over expression is an indicator of tumor aggressiveness and poor prognosis in renal cell carcinoma. J Urol 2008; 180: 1137-1140. http://dx.doi. org/10.1016/j.juro.2008.04.135

[12] HIRSCH HA, ILIOPOULOS D, JOSHI A, ZHANG Y, JAEGER SA, et al. A transcriptional signature and common gene networks link cancer with lipid metabolism and diverse human diseases. Cancer Cell 2010; 17: 348-361. http://dx.doi. org/10.1016/j.ccr.2010.01.022

[13] KUHAJDA FP. Fatty acid synthase and cancer: new application of an old pathway. Cancer Res 2006; 66: 5977-5980. http://dx.doi.org/10.1158/0008-5472.CAN-05-4673

[14] SCHAFER ZT, GRASSIAN AR, SONG L, JIANG Z, GERHART-HINES Z, et al. Antioxidant and oncogene rescue of metabolic defects caused by loss of matrix attachment. Nature 2009; 461: 109-113. http://dx.doi.org/10.1038/ nature 08268

[15] JEON SM, CHANDEL NS, HAY N. AMPK regulates NADPH homeostasis to promote tumour cell survival during energy stress. Nature 2012; 485: 661-665. http://dx.doi.org/10.1038/ nature 11066

[16] CARRACEDO A, CANTLEY LC, PANDOLFI PP. Cancer metabolism: fatty acid oxidation in the limelight. Nat Rev Cancer 2013; 13: 227-232. http://dx.doi.org/10.1038/nrc3483

[17] HARDIE DG. AMPK and autophagy get connected. EMBO J 2011; 30: 634-635. http://dx.doi.org/10.1038/emboj.2011.12

[18] HARDIE DG, ROSS FA, HAWLEY SA. AMP-activated protein kinase: a target for drugs both ancient and modern. Chem Biol 2012; 19: 1222-1236. http://dx.doi.org/10.1016/j. chembiol.2012.08.019

[19] SHI YH, DING ZB, ZHOU J, QIU SJ, FAN J. Prognostic significance of Beclin 1-dependent apoptotic activity in hepatocellular carcinoma. Autophagy 2009; 5:380-382. http:// dx.doi.org/10.4161/auto.5.3.7658

[20] SCHERZ-SHOUVAL R, ELAZAR Z. ROS, mitochondria and the regulation of autophagy. Trends Cell Biol 2007; 17: 422-427. http://dx.doi.org/10.1016/j.tcb.2007.07.009

[21] KABEYA Y, MIZUSHIMA N, UENO T, YAMAMOTO A, KIRISAKO T, et al. LC3, a mammalian homologue of yeast Apg8p, is localized in autophagosome membranes after processing. EMBO J 2000; 19: 5720-5728. http://dx.doi. org/10.1093/emboj/19.21.5720

[22] KABEYA Y, MIZUSHIMA N, YAMAMOTO A, OSHITANIOKAMOTO S, OHSUMI Y, et al. LC3, GABARAP and GATE16 localize to autophagosomal membrane depending on form-II formation. J Cell Sci 2004; 117: 2805-2812. http:// dx.doi.org/10.1242/jcs.01131

[23] SANTOS CR, SCHULZE A. Lipid metabolism in cancer. FEBS J 2012; 279: 2610-2623. http://dx.doi.org/10.1111/j.1742-4658 .2012.08644.X

[24] SCHULZE A, HARRIS AL. How cancer metabolism is tuned for proliferation and vulnerable to disruption. Nature 2012; 491: 364-373. http://dx.doi.org/10.1038/nature11706
[25] CHEONG JH, PARK ES, LIANG J, DENNISON JB, TSAVACHIDOU D, et al. Dual inhibition of tumor energy pathway by 2-deoxyglucose and metformin is effective against a broad spectrum of preclinical cancer models. Mol Cancer Ther 2011; 10: 2350-2362. http://dx.doi.org/10.1158/15357163.MCT-11-0497

[26] ROTHBART SB, RACANELLI AC, MORAN RG. Pemetrexed indirectly activates the metabolic kinase AMPK in human carcinomas. Cancer Res 2010; 70: 10299-10309. http://dx.doi. org/10.1158/0008-5472.CAN-10-1873

[27] ZHANG YL, GUO H, ZHANG CS, LIN SY, YIN Z, et al. AMP as a low-energy charge signal autonomously initiates assembly of AXIN-AMPK-LKB1 complex for AMPK activation. Cell Metab 2013; 18: 546-555. http://dx.doi.org/10.1016/j. cmet.2013.09.005

[28] HARDIE DG, ALESSI DR. LKB1 and AMPK and the cancermetabolism link - ten years after. BMC Biol 2013; 11: 36 http://dx.doi.org/10.1186/1741-7007-11-36

[29] MOTOSHIMA H, GOLDSTEIN BJ, IGATA M, ARAKI E. AMPK and cell proliferation--AMPK as a therapeutic target for atherosclerosis and cancer. J Physiol 2006; 574: 63-71. http://dx.doi.org/10.1113/jphysiol.2006.108324

[30] BAKER K, RATH T, FLAK MB, ARTHUR JC, CHEN Z, et al. Neonatal Fc receptor expression in dendritic cells mediates protective immunity against colorectal cancer. Immunity 2013; 39: 1095-1107. http://dx.doi.org/10.1016/j.immuni.2013.11.003

[31] XIANG X, SAHA AK, WEN R, RUDERMAN NB, LUO Z. AMP-activated protein kinase activators can inhibit the growth of prostate cancer cells by multiple mechanisms. Biochem Biophys Res Commun 2004; 321: 161-167. http:// dx.doi.org/10.1016/j.bbrc.2004.06.133

[32] HATZIVASSILIOU G, ZHAO F, BAUER DE, ANDREADIS C, SHAW AN, et al. ATP citrate lyase inhibition can suppress tumor cell growth. Cancer Cell 2005; 8: 311-321. http://dx.doi. org/10.1016/j.ccr.2005.09.008

[33] CORRADETTI MN, INOKI K, BARDEESY N, DEPINHO RA, GUAN KL. Regulation of the TSC pathway by LKB1: evidence of a molecular link between tuberous sclerosis complex and Peutz-Jeghers syndrome. Genes Dev 2004; 18: 1533-1538. http://dx.doi.org/10.1101/gad.1199104

[34] MIZUSHIMA N, LEVINE B, CUERVO AM, KLIONSKY DJ Autophagy fights disease through cellular self-digestion. Nature 2008; 451: 1069-1075. http://dx.doi.org/10.1038/nature06639

[35] BRENNAND A, GUALDRON-LOPEZ M, COPPENS I, RIGDEN DJ, GINGER ML, et al. Autophagy in parasitic protists: unique features and drug targets. Mol Biochem Parasitol 2011; 177: 83-99. http://dx.doi.org/10.1016/j. molbiopara.2011.02.003

[36] GILLS JJ, LOPICCOLO J, DENNIS PA. Nelfinavir, a new anti-cancer drug with pleiotropic effects and many paths to autophagy. Autophagy 2008; 4: 107-109. http://dx.doi. org/10.4161/auto.5224

[37] LIU B, CHENG Y, LIU Q, BAO JK, YANG JM. Autophagic pathways as new targets for cancer drug development. Acta Pharmacol Sin 2010;31: 1154-1164. http://dx.doi.org/10.1038/ aps.2010.118 